\title{
Hybrid Dragonfly and Particle Swarm Optimization Algorithm for Congestion Management
}

\author{
Yadu Singh \\ Pricipal \& CEO \\ KNRCE\&R Technologies \\ Hyderabad, Telangana, India
}

\begin{abstract}
The transmission line undergoes large disadvantages of insecurity as well as discrete power flows regarding the rescheduling cost. In the Transmission line, an optimal solution for this discrete as well as secure power flow is attained from the Real Power Balance Constraint (RBCM) method. To produce a limited solution a large number of optimization approaches is used for congestion management. In this work, a highly powerful method as the Hybrid Dragonfly and PSO algorithm named (DA-PSO) with the main contribution to resolve the cost of congestion with minimizing rescheduled cost. Furthermore, to identify the improvement in the developed approach performance regarding congestion an analysis is performed among the developed and existing techniques in order to fulfill the analysis. The performance analysis examination outcome exhibit that the developed model is highly superior to the conventional models regarding the reduced rescheduling cost.
\end{abstract}

Keywords: Congestion Management, Optimization Algorithm, Power Flow, Rescheduling Cost, Transmission Line.

\begin{tabular}{ll} 
Nomenclature & \\
\hline Abbreviations & Descriptions \\
\hline ACO & Ant Colony Optimization \\
CAISO & California ISO \\
GA & Genetic Algorithm \\
RUL & Ramp up Limits \\
SA & Simulated Annealing \\
DESS & Distributed Energy Storage System \\
DLFU & Disparity Line Utilization Factor \\
APO & Active Power Output \\
PSO & Particle Swarm Optimization \\
FACTSs & Flexible AC transmission systems \\
GCC & Generation Capacity Constraint \\
OPF & Optimal Power Flow \\
PV & Photo voltaic \\
DG & Distributed Generator \\
SL & Stability Limits \\
GSA & Gravitational Search Algorithm \\
ITVAC & Improved Time-Varying Acceleration Coefficients \\
RPBC & Real Power Balance Constraint \\
ESS & Energy Storage System \\
TCSCs & Thyristor Controlled Series Compensators \\
DE & Differential Evolution \\
\hline
\end{tabular}

\section{Introduction}

The operation manner, ownership, structure, and utilities management are change by the deregulated electric power industries. In deregulated as well as competitive markets, the transmission congestion problem is highly well-known which requires a suitable management scheme [1]. For the electric uses, it is very important to operate in the new competitive electric market so that it creates superior 
consumption of the conventional transmission facilities in combination to maintain the stability, security as well as supplied power reliability. For the conventional transmission lines, transmission facilities, lie to obtain congested or overloaded in the procedure of superior use. In the transmission lines, raised congestion might tend to an incident in the network. An uncontrolled contingencies series is represented as the important cause of blackouts. Therefore, to appropriately use the transmission lines as well as at a similar time the stability, security well as reliability is maintained of the transmission system, the FACTS devices employ turns out to be predictable. The number of FACTS devices, which can be exploited in the economic constraints put a restriction. Therefore, the optimal positioning and the FACTS devices tuning in the power system are obligatory [2].

Because of deregulation, the prologue of competition may cause the cost-based electricity to transfer into a price-based market product [3]. The net electricity cost is reduced by the raised competition and the market forces are driven by electricity prices. In new liberalized market competition, may cause selfdetermining endangering usefulness to sell all their produced power to the customers [12]. Therefore they attempt to contain all their produced power on the transmission line that might occur transmission line infringement limits namely voltage limit, thermal limit, and stability limit so on therefore occurs the congestion in a transmission line. The congestion in transmission line might tend to overload lines tripping, instability of power system so on, also apparently raise the electricity cost as it happens the power system to diverge from its optimal operation. Therefore, the congestion requires to ease rapidly[4].

To safeguard the system from serious circumstances, congestion management is exploited [9]. It is given maximum precedence by CAISO and FERC. Moreover, for congestion management, several techniques were exploited namely DE, OPF, and PSO [10]. In an electrical network, FACTS positioning significantly improves the power transfer ability of lines and networks. The FACTS devices are considered as the solid stated converters that control several electrical parameters in the transmission system as well as improve the stability of the power system. Numerous approaches were proposed until now to conflict congestion namely transmission line switching, generation rescheduling [11].

To optimize a non-linear function, numerous existing optimization approaches namely the interior point approach, Newton's approach, and gradient approach so on are present in state-of-art. As aforesaid existing techniques are iterative in nature and their search direction is ascertained from function derivative, hence it turns out to be essential to state the objective model in the type of continuous differential function. To override this issue, in the current day's meta-heuristics techniques namely evolutionary approach, GA, tabu search, ACO, SA, PSO so on are introduced. As PSO is a computational intelligence-based optimization approach that is not highly pretentious by optimization issue and size nonlinearity as well as it can converge to the optimal solution in numerous issues whereas largely analytical techniques fail to converge, thus it can be powerfully used for various optimization issues in power systems.

The major contribution of this research is to propose a new CM model with the aspiration of delimiting the power system using Advanced Soft Computing Models. The main idea behind the generation rescheduling is used even if there subsist a count of congestion Management techniques. Moreover, it is attained using an optimization approach called Hybrid DA-PSO to resolve the objective function.

\section{Literature Review}

In 2009, Hadi Besharat and Seyed Abbas Taher [1], worked on a deregulated electricity market. The enduring restructuring of the power system needs an opening of unemployed potentials of the transmission system. It was because of environmental, main concern, and cost issues that were the most important obstacles for expansion of power transmission network. To minimize the flows in heavily loaded lines, FACTSs devices were an option, ensuing in a minimum system loss, maximized loadability, minimized production cost, enhanced network stability, as well as a satisfied contractual obligation by controlling the power flows in the network. Here, an approach to establish the optimal TCSCs position was recommended based on minimization of total system VAR power losses and performance index of real power. In 2017, Akanksha Mishra and Venkata Nagesh Kumar [2], worked on a DLFU for optimal positioning as well as GSA-based optimal tuning of IPFC for the congestion control in transmission lines. Regarding the relative line congestion, the DLFU ranks the transmission lines. In the majority congested line, the IPFC was positioned as well as in the minimum congested line was linked to a similar bus. In order to tune the IPFC parameters, a multi-objective function was chosen. In 2020, Divya Asija et al [3], worked on the Solar PV beside with ESS was exploited as DESS. During off-load period to accumulate excess energy; ESS beside DG was exploited that was used at peak load thus improving the complete system effectiveness. In 2016, Md Sarwar and Anwar Shahzad Siddiqui [4], worked on a technique to deal with congestion in the deregulated environment by exploiting the PSO-ITVAC. On the basis of the generator sensitivities magnitude, the congestion was lessened by selecting the optimally rescheduling the 
generator's active power output. By exploiting the PSO-ITVAC the rescheduling cost was reduced. In 2012, Nima Amjady and Mahmood Hakimi [5], worked on a novel congestion management model taking into consideration of power system dynamic voltage stability boundary. Therefore this reason, accurate dynamic modeling of power system equipment, such as loads as well as generators, was integrated into the developed CM model. The developed technique mitigates congestion with a minor cost of CM and more dynamic voltage stability margin, ensuing in a highly robust power system, evaluated to the preceding CM approaches.

\section{Congestion Management Formulation in Deregulated Power System}

\subsection{Congestion Cost}

In the electrical environment, congestion can be minimized using the generator's APO rescheduling. The process of rescheduling can be attained, by falling or expanding in the APO. This reduction or deaccelerating or in the cost of APO requirements as well as this cost is present regarding the price bids that are being submitted using GENCOs. For scheduling cost incurred, is indicates as the cost of congestion as well as the mathematical formulation of congestion cost is referred to as in eq. (1) in that the cumulative cost needed to modify APO is indicated regarding $(\$ / n)$ is stated as $C_{\text {total }}$ and $C_{j}: j=\mathbf{1 , 2}, \ldots, N_{g}$. To expand

the price bids and power is referred to as $D_{j}$ indicated regarding $\$ / \mathrm{MWn}$ by $j^{\text {th }}$ GENCO. $\Delta \mathrm{P}_{\mathrm{G}}^{+}(\mathrm{j})$ and $\Delta \mathrm{P}_{\mathrm{G}}(\mathrm{j})$ represented as AP expanded and generator decrement (MW). $\Delta \mathrm{P}_{\mathrm{G}}^{+}(\mathrm{j})$ as well as $\Delta \mathrm{P}_{\mathrm{G}}(\mathrm{j})$ states the change in AP. In AP the change is stated as the "absolute difference amid price bids submitted at the present hour (n) from the preceding hour (n-1)" and the mathematical formulation of change in AP is stated as $\Delta P_{G}$, is exhibited in Eq. (2). Here, the AP produced at the preceding hour and the current hour are shown as $P_{G}(n)$ and, $\mathrm{P}_{\mathrm{G}}(\mathrm{n}-\mathbf{1})$ correspondingly.

$$
\begin{aligned}
& \mathrm{C}_{\text {total }}=\sum_{j \in N_{g}}\left(C_{j} \Delta P_{G}^{+}(j)+D_{j} \Delta P_{G}(j)\right) S / n \\
& \Delta P_{G}=\left|P_{G}(n-1)-P_{G}(n)\right|
\end{aligned}
$$

\subsection{Constraints}

To overrule the issue of congestion, the rescheduling scheme is used with low minimum rescheduling cost. The constraints namely GCC, RPBC, SL, and RUL are exploited at the time of the rescheduling operation of the power generators. Hence, it is necessary to model a minimum rescheduling cost based on the Constrained Minimization Function (CMF). For $G^{\text {th }}$ generation unit, the change in the AP generated using MW is signified as $\Delta \mathrm{P}_{\mathrm{G}}$ whereas, $\Delta \mathrm{P}_{\mathrm{G}}: \mathbf{0} \leq \mathrm{j} \leq \mathrm{N}_{\mathrm{g}}(\mathrm{MW})$ for a cost of $\mathrm{f}\left(\Delta \mathrm{P}_{\mathrm{G}}\right)$ regarding represented concerning $\$$. The minimization function is stated in eq. (3).

$$
\mathrm{s}^{*}=\underset{\Delta \mathrm{P}_{\mathrm{G}} \forall \mathrm{G}}{\arg } \min \sum_{\mathrm{j}=\mathbf{1}}^{\mathrm{N}_{\mathrm{g}}} \mathrm{f}\left(\Delta \mathrm{P}_{\mathrm{G}}(\mathrm{j})\right)
$$

Furthermore, Eq. (I3) experiences the following constraints

\section{a) GCC:}

The AP quantity which is produced regarding $\mathrm{MW}$ is simplified as $\mathrm{P}_{\mathrm{G}}$ is stated in eq. (4). $\mathrm{P}_{\mathrm{G}}^{\max }$ referred to as the maximum quantity produced in $\mathrm{MW}$, whereas $\mathrm{P}_{\mathrm{G}}^{\mathrm{min}}$ represents the least quantity generated in $\mathrm{MW}$. The $\mathrm{P}_{\mathrm{G}}$ value is estimated on the basis of eq. (5), and the power quantity which is being produced is signified as $\Omega P_{\mathrm{G}}$.

$$
\begin{aligned}
& \mathrm{P}_{\mathrm{G}}^{\min } \leq \mathrm{P}_{\mathrm{G}} \leq \mathrm{P}_{\mathrm{G}}^{\max } \\
& \mathrm{P}_{\mathrm{G}}=\Omega \mathrm{P}_{\mathrm{G}}+\Delta \mathrm{P}_{\mathrm{G}}
\end{aligned}
$$




\section{b) RPBC}

In eq. (6), $\mathrm{P}_{\mathrm{TL}}$ indicates the transmission loss in $\mathrm{MW}$ and $P_{P D}$ indicates the power demand. For transmission loss $P_{T L}$ mathematical formulation is stated in eq. (7), where the loss coefficients are $\mathrm{C}_{\mathrm{ij}}, \mathrm{C}_{\mathbf{0 j}}, \mathrm{C}_{\mathbf{0 0}}$.

$$
\begin{aligned}
& \sum_{j=\mathbf{1}}^{N_{g}} P_{G}-\left(P_{P D}+P_{T L}\right)=\mathbf{0} \\
& P_{T L}=\sum_{i=1}^{N_{g}} \sum_{j=1}^{N_{g}}\left(P_{i} C_{i j} P_{G}\right)+\sum_{j=1}^{N_{g}}\left(C_{0 j} P_{G}\right)+C_{00}
\end{aligned}
$$

c) SL

The sequential stability limits are exploited to explain and to assure the congestion alleviation stated. From the generator, the voltage stability is explained regarding the angle limits $\left(\partial_{G}^{\min }, \partial_{G}^{\max }\right)$ as well as voltage limits $\left(V_{G}^{\min }, V_{G}^{\max }\right)$. In MV and MPFL the power flow is signified as $P_{G}^{2}, Q_{G}^{2}$ and $S_{G}^{\max }$, correspondingly.

$$
\begin{aligned}
& \mathrm{V}_{\mathrm{G}}^{\min } \leq \mathrm{V}_{\mathrm{G}} \leq \mathrm{V}_{\mathrm{G}}^{\max } \\
& \partial_{\mathrm{G}}^{\min } \leq \partial_{\mathrm{G}} \leq \partial_{\mathrm{G}}^{\max } \\
& \mathrm{P}_{\mathrm{G}}^{\mathbf{2}}+\mathrm{Q}_{\mathrm{G}}^{\mathbf{2}} \leq\left(\mathrm{S}_{\mathrm{G}}^{\max }\right)^{\mathbf{2}}
\end{aligned}
$$

\section{d) RUL}

RUL is represented as "up as well as down quantity limits of cost of rescheduling". The ramp limits are explained to create the rescheduling costs to turn out to be equal up and down.

$$
\Delta \mathrm{P}_{\mathrm{G}}^{\min } \leq \Delta \mathrm{P}_{\mathrm{G}} \leq \Delta \mathrm{P}_{\mathrm{G}}^{\max }
$$

In the least quantity generated the change in MW is $\Delta P_{G}^{\min }$ and $\Delta P_{G}^{\max }$ represents the change in the utmost quantity produced in MW. From Eq. (3), to convene the aforesaid constraints the optimal rescheduling scheme $S^{*}$ is derived.

\section{e) Objective Function}

The cost of $f\left(\Delta \mathrm{P}_{\mathrm{g}}\right)$ represents the summation total of the cumulative cost acquired for rescheduling as well as violation level on the voltage of equivalent TL, for $\mathrm{j}^{\text {th }}$ generation unit. To describe RS, eq. (12) shows the cost function. The penalty cost which is used on violating the constraints is stated as $\mathrm{P}_{\text {constraints }}$ and $\mathrm{P}_{\text {profile }}$ represents the penalty cost forced on voltage profile. On the basis of eq. (13), the $\mathrm{C}_{\text {profile }}$ value can be attained.

$$
\begin{aligned}
& \mathrm{f}\left(\Delta \mathrm{P}_{\mathrm{g}}\right)=\mathrm{C}_{\text {total }}+\mathrm{P}_{\text {profile }} \mathrm{C}_{\text {profile }}+\mathrm{P}_{\text {constra int s }} \mathrm{C}_{\text {constra int }} \\
& \mathrm{C}_{\text {profile }}=\left|\min \left(\mathrm{V}_{\mathrm{g}}-\mathrm{V}_{\mathrm{g}}^{\min }, \mathbf{0}\right)\right|+\left|\min \left(\mathrm{V}_{\mathrm{g}}^{\max }-\mathrm{V}_{\mathrm{g}}, \mathbf{0}\right)\right|
\end{aligned}
$$

\section{Proposed Hybrid DA-PSO Algorithm for Congestion Management 4.1 Conventional Dragonfly Algorithm}

DA is enthused by the unique as well as better dragonflies' swarming behavior [6]. The migration, as well as hunting, is the behavior of the dragonfly swarms.Let us state dragonflies population size is $\mathrm{N}$. The location of $i^{\text {th }}$ dragonfly is stated in Eq. (14)

$$
\mathrm{X}_{\mathrm{i}}=\left(\mathrm{x}_{\mathrm{i}}^{1}, \mathrm{x}_{\mathrm{i}}^{\mathrm{d}}, \ldots \ldots, \mathrm{x}_{\mathrm{i}}^{\mathrm{N}}\right)
$$

Whereas $i=\mathbf{1}, \mathbf{2}, \mathbf{3}, \ldots, N, N$ states count of search agents, $x_{i}^{d}$ states location of the $i^{\text {th }}$ dragonfly in $d^{\text {th }}$ search space dimension. 
On basis of the initial location values, the fitness function is estimated that is arbitrarily produced amid the upper and lower bounds of variables. $S$ indicates the weight for separation, c indicates cohesion(c), $a$ indicates alignment, $\mathrm{f}$ indicates food, and $\mathrm{e}$ indicates enemy factors for each dragonflywhich is initialized arbitrarily. In order to update the velocity, as well as location of dragonflies factors, are computed based on eq. (15) to (17).

$$
\begin{aligned}
& S_{i}=-\sum_{j=1}^{N} X-X_{i} \\
& A_{i}=\frac{\sum_{j=1}^{N} V_{i}}{N} \\
& C_{i}=\frac{\sum_{j=1}^{N} X_{i}}{N}-X
\end{aligned}
$$

whereas $V_{i}$ and $X_{i}$ states velocity and position of the $i^{\text {th }}$ individual. $N$ indicates a number of neighboring individuals and $V_{i}$ indicates the current individual location. $F_{i}$ indicates the Attraction towards a food source, $E_{i}$ indicates the distraction from enemies, and its calculation is stated in eq. (18) and (19).

$$
\begin{aligned}
& F_{i}=X^{+}-X \\
& E_{i}=X^{-}+X
\end{aligned}
$$

Whereas, $\mathrm{X}$ signifies current individual position, $\mathrm{X}^{-}$signifies enemy source as well as $\mathrm{X}^{+}$signifies the food source.

The neighborhood distance is computed using the Euclidean distance amid all the dragonflies as well as choosing $\mathrm{N}$ of them. $\mathrm{r}_{\mathrm{ij}}$ indicates distance, which is computed using Eq. (20).

$$
\mathrm{r}_{\mathrm{ij}}=\sqrt{\sum_{\mathrm{k}=1}^{\mathrm{d}}\left(\mathrm{x}_{\mathrm{i}, \mathrm{k}}-\mathrm{x}_{\mathrm{j}, \mathrm{k}}\right)^{2}}
$$

On the basis of Eq. (21) analogous to velocity formulation of PSO as well as dragonfly location will be updated based on Eq. (22) that is analogous to the location formulation of PSO.

$$
\begin{aligned}
& \Delta X_{t+1}=\left(s_{i}+a A_{i}+c C_{i}+f_{i}+e E_{i}\right)+w \Delta X_{t} \\
& X_{t+1}=X_{t}+\Delta X_{t+1}
\end{aligned}
$$

In the neighborhood, if there is no dragonfly radius dragonfly location will be updated exploiting Levy Flight [8] equation which is stated in Eq. (23). This enhances the arbitrariness, as well as global search capability chaotic behavior of dragonflies.

$$
\mathrm{X}_{\mathrm{t}+\mathbf{1}}=\mathrm{X}_{\mathrm{t}}+\operatorname{levy}(\mathrm{d}) \mathrm{X}_{\mathrm{t}}
$$

The fitness function is subsequently calculated on the basis of the updated velocities as well as location.

\subsection{Conventional Particle Swarm Optimization Algorithm}

PSO is a swarm intelligence optimization approach based on swarms social behavior [7]. Let $\mathrm{N}$ be dimensional search space. Consider velocity as well as the position of $\mathrm{i}^{\text {th }}$ particle in $\mathrm{k}^{\text {th }}$ iteration be $\mathrm{X}_{\mathrm{k}}^{\mathrm{i}}$ and velocity $\mathrm{V}_{\mathrm{k}+1}^{\mathrm{i}}$ correspondingly. The location and velocity of a particle in $(k+1)$ iteration are updated as per Eq. (24) and (25) correspondingly.

$$
\begin{aligned}
& \mathrm{V}_{\mathrm{k}+\mathbf{1}}^{\mathrm{i}}=\mathrm{wV}_{\mathrm{k}+\mathbf{1}}^{\mathrm{i}}+\mathrm{C}_{\mathbf{1}} \mathrm{r}_{1}\left(\mathrm{P}_{\mathrm{k}}^{\mathrm{i}}-\mathrm{X}_{\mathrm{k}}^{\mathrm{i}}\right)+\mathrm{C}_{2} \mathrm{r}_{2}\left(\mathrm{P}_{\mathrm{k}}^{\mathrm{g}}-\mathrm{X}_{\mathrm{k}}^{\mathrm{i}}\right) \\
& X_{k+1}^{i}=X_{k}^{i}+V_{k+1}^{i}
\end{aligned}
$$

whereas $\mathrm{C}_{\mathbf{1}}$ indicates cognitive parameters and $\mathrm{C}_{\mathbf{2}}$ indicates social parameters, $w$ indicates the inertial weight $\mathrm{P}_{\mathrm{k}}^{\mathrm{i}}$ and $\mathrm{P}_{\mathrm{k}}^{\mathrm{g}}$ represents the pbest of the $i$ th particle. 


\subsection{Proposed Hybrid DA-PSO}

An appropriate balance amid exploitation as well as exploration of search space is essential to attain a global optimal solution for any optimization approach. The exploitation or else called intensification engages search in a local region based on the present optimal solution and exploration or else called diversification engages global search in search space. In excess of exploitation and exploration destructively affect the approach performance by rising the convergence time as well as raising the probability to fall into local optima.

The traditional DA operates on an arbitrarily produced initial population of search agents as well as by exploiting Levy flight dragon flies explore the search space.

This arbitrary initialization as well as levy flight search procedure raises solution diversity and makes stronger the exploration ability of approach. Furthermore, DA possess merely some parameters to regulate as well as adaptive tuning of these swarming factors aids in balancing global and local search abilities.

Nevertheless,DA is short of an internal memory that can keep track of before attained possible solutions. At the time of the procedure, DA evades all the fitness values more than global optimal and by no means stays path on probable set of solutions that can converge to global optima. This weakens the exploitation ability of the DA attention to converge gradually and now and then idle at local optima. In order to evade this, a new hybrid approach on the basis of the PSO and DA is developed.

Here, 2 features are augmented to the existing DA approach to manage its performance, such as a) an internal memory to stay track of probable solutions that have a possible to converge to global optima b) hybrid with PSO in iteration level that runs on this set of stored solutions.

The velocity as well as position formulation of PSO is enhanced as

$$
\begin{aligned}
& V_{k+1}^{i}={ }^{i} V_{k+1}^{i}+C_{1} r_{1}\left(D A-\text { Pbest }_{k}^{i}-X_{k}^{i}\right)+C_{2} r_{2}\left(D A-\text { gbest }_{k}^{g}-X_{k}^{i}\right) \\
& X_{k+1}^{i}=X_{k}^{i}+V_{k+1}^{i}
\end{aligned}
$$

\section{Simulation Setup}

In this section, the experimental evaluation of the proposed technique was demonstrated. Here, IEEE 14 and 30 bus system was utilized as the benchmark test bus system to perform the experimentation analysis. Moreover, within the system, the TLs, CBs, and loads as well as generators were exploited. In IEEE 14 bus system, 3 GENCOS were used and in IEEE 14 bus system, 6 GENCOS were exploited in the IEEE 30 bus system. Moreover, the performance evaluation was performed on the basis of the cost of congestion, cost of compensation as well as final cost for both the developed and conventional models. Here, the proposed method was compared with the conventional models such as Artifical Bee Colony (ABC), Genetic Algorithm (GA) and Firefly (FF).

Fig 1 demonstrates the analysis of adopted as well as traditional techniques regarding congestion cost for both the IEEE 14 and IEEE 30 bus system. The analysis of the proposed and conventional models regarding the compensation cost for both the IEEE 14 as well as the 30 bus system is illustrated in Fig 2 . Fig 3 shows an analysis of adopted as well as traditional techniques regarding the final cost for both the IEEE 14 as well as 30 bus system.

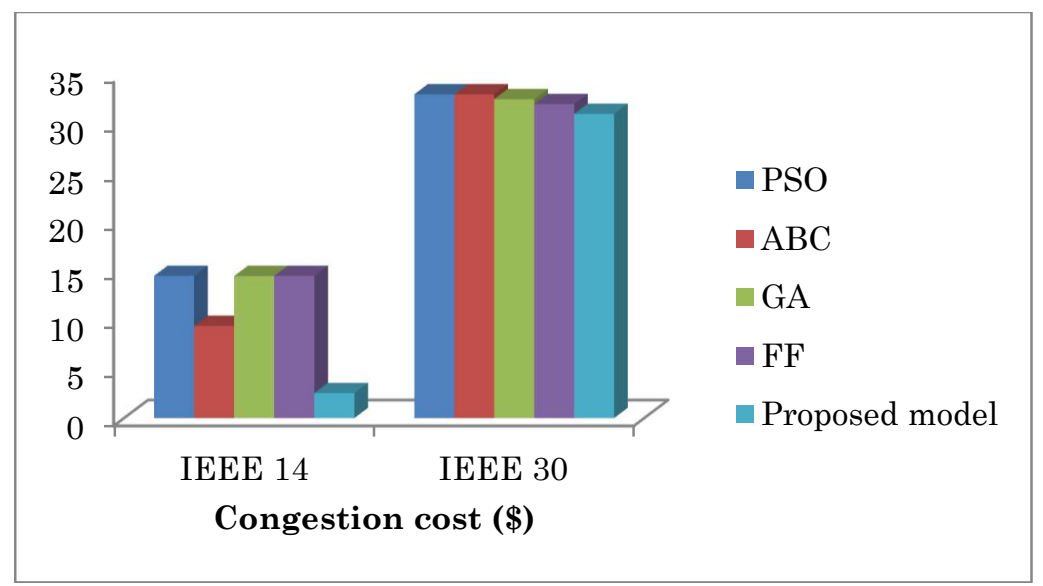

Fig. 1 Performance analysis of the adopted as well as traditional approaches concerning congestion cost 


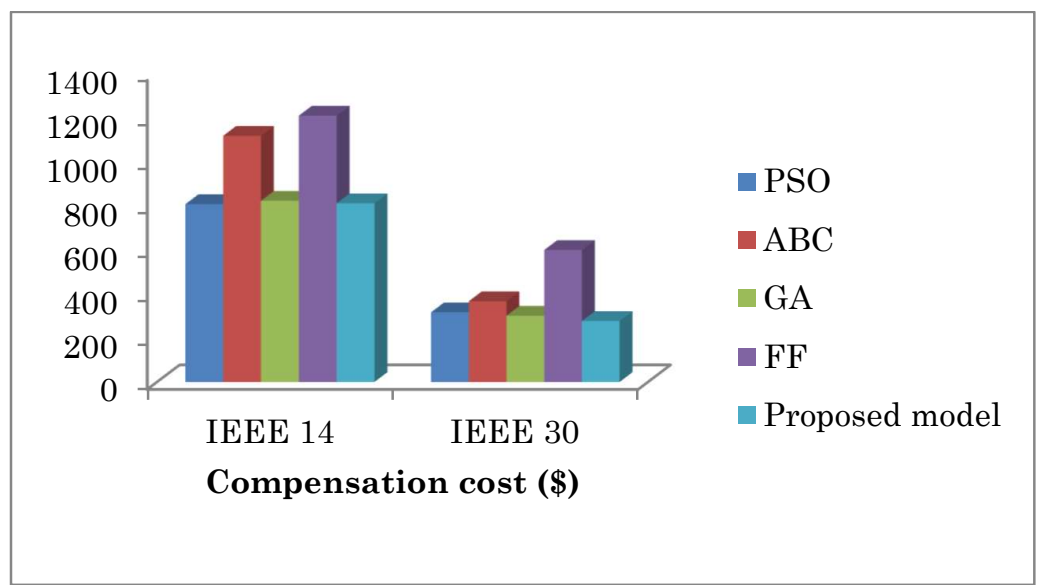

Fig. 2 Performance analysis of adopted as well as traditional approaches concerning compensation cost

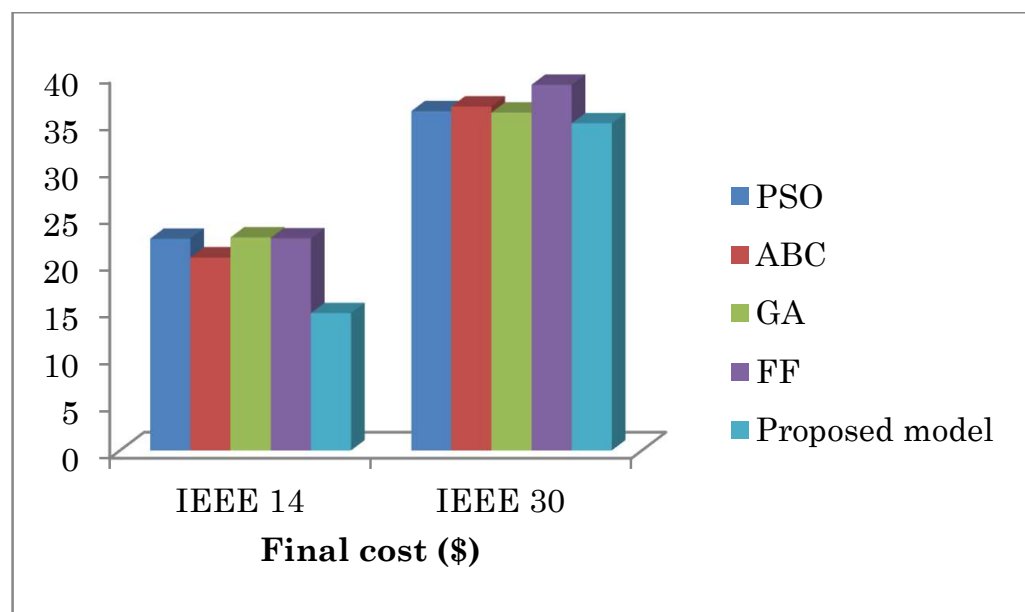

Fig. 3 Performance analysis of the adopted as well as traditional approaches concerning the final cost

Table 1 Statistical analysis of the developed and traditional techniques for IEEE 14 Bus System

\begin{tabular}{llllll} 
Techniques & Best & Mean & Std & Worst & Median \\
\hline PSO & 21.641 & 21.666 & 1.11872 & 21.687 & 21.66 \\
ABC & 14.627 & 21.972 & 3.647 & 22.686 & 22.661 \\
GA & 21.644 & 21.372 & 1.1184 & 22.616 & 21.664 \\
FF & 22.684 & 22.697 & 1.11672 & 22.726 & 22.691 \\
Proposed model & 14.614 & 18.166 & 3.2412 & 21.648 & 21.621 \\
\hline
\end{tabular}

Table 2 Statistical analysis of the proposed and conventional models for IEEE 30 Bus System

\begin{tabular}{llllll}
\hline Techniques & Worst & Median & Std & Best & Mean \\
\hline PSO & 27.99 & 27.156 & 0.26267 & 27.044 & 27.222 \\
ABC & 27.901 & 27.759 & 0.10652 & 27.759 & 27.707 \\
GA & 27.002 & 25.659 & 0.07222 & 25.659 & 25.697 \\
FF & 26.976 & 26.767 & 0.17102 & 26.552 & 26.769 \\
Proposed model & 25.66 & 25.659 & 0.00927 & 25.659 & 25.672 \\
\hline
\end{tabular}

The statistical analysis is performed in the obtained results to evaluate the conventional techniques for IEEE 14 and IEEE 30 bus system and it is summarized in Table 1 and 2.

\section{Conclusion}

The rescheduling of generation was utilized in this paper with the main objective of presenting the security to the power system and continual power supply with short of congestion in the TL. The generator was considered as one of the Congestion Management techniques as well it possesses confronts of reducing the cost of rescheduling. Therefore, the cost of rescheduling was reduced using an optimized approach in this work, as well as a performance-based evaluation was performed amid the proposed as 
well as conventional techniques. Here, the performance analysis was performed regarding the congestion and cost. Therefore, the overall analysis states that the performance of the developed technique was superior to the conventional methods regarding the reduced cost of congestion as well as compensation cost.

\section{Compliance with Ethical Standards}

Conflicts of interest: Authors declared that they have no conflict of interest.

Human participants: The conducted research follows the ethical standards and the authors ensured that they have not conducted any studies with human participants or animals.

\section{References}

[1] Hadi BesharatSeyed Abbas Taher,"Congestion management by determining optimal location of TCSC in deregulated power systems",International Journal of Electrical Power \& Energy Systems, vol.30, no. 10, December 2008.

[2] Akanksha MishraVenkata Nagesh Kumar G,"Congestion management of deregulated power systems by optimal setting of Interline Power Flow Controller using Gravitational Search algorithm", Journal of Electrical Systems and Information Technology, vol. 4, no.1, pp.198-212, 19 October 2016.

[3] Divya AsijaPallavi Choudekar,"Congestion management using multi-objective hybrid DE-PSO optimization with solar-ess based distributed generation in deregulated power Market", Renewable Energy Focus, vol. 36, pp.3242, 4 November 2020.

[4] Divya AsijaPallavi Choudekar,"Congestion management using multi-objective hybrid DE-PSO optimization with solar-ess based distributed generation in deregulated power Market",Renewable Energy Focus, vol.36, pp. 32-42, 4 November 2020.

[5] Nima AmjadyMahmood Hakimi,"Dynamic voltage stability constrained congestion management framework for deregulated electricity markets", Energy Conversion and Management, vol.58, pp.66-75, June 2012.

[6] A. Sunny KumarT. V. Hanumantha RaoR. T. RamaKanth,"Optimizing pulsed current micro plasma arc welding parameters to maximize ultimate tensile strength of titanium (Ti-6Al-4V) alloy using Dragon fly algorithm", Materials Today: Proceedings24 October 2019.

[7] A. RajeshP. Ashoka VarthananP. V. Suchith,"Optimization of shot peening process parameters using PSO algorithm to maximise the fatigue strength, flexural strength and surface hardness of AA2024-T3 alloy", Materials Today: ProceedingsAvailable online 22 February 2021.

[8] Yang, X.-S.(2010a). Firefly algorithm, lévy flights and global optimization. In M. Bramer, R. Ellis, \& M. Petridis (Eds.), Research and development in intelligent systems XXVI: Incorporating applications and innovations, in intelligent systems XVII. London: Springer London, pp. 209-218,2010.

[9] T C Srinivasa Rao,S S Tulasi Ram and J B V Subrahmanyam,"Fault Recognition and Diagnosis Based on a Decision Tree for Power Distribution Systems",Journal of Computational Mechanics, Power System and Control (JCMPS), vol. 2, no. 2, April 2019

[10] V.Tejaswini and Dr.D.Susitra,"Hybrid PSO-WOA for Solving ORPD Problem under Unbalanced Conditions",Journal of Computational Mechanics, Power System and Control (JCMPS), vol. 2, no. 2, April 2019.

[11] Mr. Rupam Gupta Roy,"Economic Dispatch Problem in Power System Using Hybrid PSO and Enhanced Bat Optimization Algorithm", Journal of Computational Mechanics, Power System and Control (JCMPS), vol. 3, no. 3, July 2020.

[12] K.Srinivas,"Prediction of E-Learning Efficiency by Deep Learning in EKhool Online Portal Networks",Multimedia Research, vol.3, no. 4, October 2020. 